\title{
Melatonin and Circadian Rhythm: Aging, Cancer, and Mechanism
}

\author{
Peter Kovacic ${ }^{1 *}$, Ratnasamy Somanathan ${ }^{1,2}$ \\ ${ }^{1}$ Department of Chemistry and Biochemistry, San Diego State University, San Diego, USA \\ ${ }^{2}$ Centro de Graduados e Investigación del Instituto Tecnológico de Tijuana, Tijuana, Mexico \\ Email: *pkovacic@mail.sdsu.edu
}

Received 25 May 2014; revised 27 June 2014; accepted 17 July 2014

Copyright (C) 2014 by authors and Scientific Research Publishing Inc.

This work is licensed under the Creative Commons Attribution International License (CC BY). http://creativecommons.org/licenses/by/4.0/

(c) (7) Open Access

\begin{abstract}
The circadian clock is involved with many aspects of biological processes in animals and plants. Melatonin is a pineal hormone which is known to play a widespread role in the biochemistry of the circadian clock. The present review addresses the clock and melatonin in relation to aging and cancer, which are important aspects of medicine and biochemistry. The literature contains a large variety of associations of these areas with melatonin and circadian rhythm. Mechanistic aspects are addressed with emphasis on the unifying theme based on electron transfer-free radicalsoxidative stress.
\end{abstract}

Keywords

Melatonin, Circadian Clock, Aging, Cancer, Mechanism

\section{Introduction}

This review presents a multifaceted approach to melatonin and circadian rhythm in relation to cancer and aging. The circadian clock, which likely evolved 2.5 billion years ago, has been the object of attention in science and the media [1]. The clock, which tracks time in 24 hour cycles, is controlled by genes in cycling feedback loops. An important feature is metabolic efficiency which is promoted by timing activities with day-night rhythms. The machinery plays a role not only in sleep cycles, but also in health and disease. Adverse medical events that relate to rhythm dysfunction are cancer, heart disease, addiction, diabetes and obesity.

This review deals with cancer, aging, and circadian rhythm [2] which are important aspects of medicine and biochemistry, in relation to melatonin. Genes play a fundamental role in many areas of human biology. Mechanistic aspects of the various topics are also treated. The role of melatonin, a pineal hormone, is addressed. The preponderance of bioactive substances, usually as their metabolites, incorporates ET functionalities. We believe that it plays an important role in physiological responses. The main groups include quinones (or phenolic pre-

*Corresponding author. 
cursors), metal complexes (or complexors), aromatic nitro compounds (or reduced hydroxylamine and nitroso derivatives), and conjugated imines (or iminium species). In vivo redox cycling with oxygen can occur, giving rise to oxidative stress (OS) through generation of reactive oxygen species (ROS), such as hydrogen peroxide, hydroperoxides, alkyl peroxides, and diverse radicals (hydroxyl, alkoxyl, hydroperoxyl, and superoxide). In some cases, ET results in involvement with normal electrical effects (e.g., in respiration or neurochemistry). Generally, active entities possessing ET groups display reduction potentials in the physiologically responsive range, (i.e., more positive than about $-0.5 \mathrm{~V}$ ). Hence, ET in vivo can occur resulting in production of ROS which can be beneficial in cell signaling at low concentrations, but produce toxic results at higher levels. Electron donors consist of phenols, $\mathrm{N}$-hetercycles or disulfides in proteins which can produce relatively stable radical cations. ET, ROS, and OS have been increasingly implicated in the mode of action of drugs and toxins.

There is a plethora of experimental evidence supporting the ET-ROS theoretical framework. The evidence includes the generation of the common ROS, lipid peroxidation, degradation products of oxidation, depletion of Ass, effect of exogenous AOs, and DNA oxidation and cleavage products, as well as electrochemical data. This comprehensive, unifying mechanism is in consistent with the frequent observations that many ET substances display a variety of activities (e.g., multiple-drug properties), as well as toxic effects.

It is important to recognize that mode of action in the biodomain is often multifaceted. In addition to the ET-ROS-OS approach, other aspects may pertain, such as enzyme inhibition, allosteric effects, receptor binding, metabolism and physical factors.

\section{Melatonin}

Melatonin is an internal factor affecting the circadian clock. In all vertebrates investigated so far, melatonin is produced in the pineal body and resynchronizes the SCN by providing information about light/darkness from the retinohypothalmic tract. Melatonin is also essential for regulation of rhythmic functions in peripheral target tissues of the clock. It peaks during darkness and lowers during the day; several reviews have appeared over the years on this subject [3]-[5]. Though this indole hormone was first reported from the pineal gland, it was also found in retina, Harderian gland and gastro-intestinal tracts in vertebrates, as well as in a wide variety of organisms, ranging from invertebrates to plants [5]. One of the characteristic features of the tryptophan derivative molecule is that its synthesis takes place during darkness in a light-dark cycle, irrespective of the habit of the animal. Therefore, it is often described as "hormone of darkness" or a "chronobiotic molecule". In all vertebrates investigated so far, melatonin is primarily synthesized within the pinealocytes of the pineal gland during the night, regardless of the diurnal or nocturnal locomotor activity of the animals [5].

Biosynthesis of melatonin is a four-step phenomenon [3]. First, its precursor L-tryptophan is taken from the circulation (blood) into pinealocyte and converted to 5-hydroxytryptophan by tryptophan-5-monooxygenase/ hydrolase and further decarboxylated by aromatic amino acid carboxylase to form 5-hydroxytryptamine or serotonin. Seratonin is acetylated ( $\mathrm{N}$-acylation) to form $\mathrm{N}$-acetylseratonin by serotonin-N-acetyltransferace. Finally, $\mathrm{N}$-acetylseratonin is methylated by hydroxyindole-O-methyltransferase to form melatonin [5] (Scheme 1).

The rhythm of melatonin synthesis is generated by interacting networks of circadian clock genes located in the SCN, circadian oscillator/master pacemaker of the hypothalamus in brain, which is considered as the major central rhythm-generating system or "clock" in mammals [6] [7]. The SCN clock is set to a 24 h day by the natural light-dark cycle via retinal light input which then sends circadian signals over a neural pathway including sympathetic nerve terminals that project from the superior cervical ganglia (SCG) to the pineal gland and thereby driving rhythmic melatonin synthesis [4] [5]. The main photoreceptor pigment for circadian timing appears to be melanopsin in the retinal ganglion cells [8]. SCN is the major regulatory site of serotonin N-acetyl transferase (NAT) activity, which is the key enzyme in the synthesis of melatonin from tryptophan. Melatonin, through various receptors (MT1, MT2 and MT3) induces a differential influence on clock genes [4] [5]. In humans, the pars tuberalis and SCN clock genes expression pattern show $24 \mathrm{~h}$ rhythmicity. The synthesis of melatonin is driven by the SCN to convey photoperiodic information to the pars tuberalis, which in turn influences the clock genes PER1 and Cry 1 within the pars tuberalis, translating the melatonin signal to control body rhythm [4] [5].

Melatonin involvement with circadian rhythm and other aspect is related to recent articles [9] [10].

Structure activity relationship (SAR) studies were carried out on melatonin [11]. Data indicate the crucial role played by the methoxy substituent. Various investigations were carried out on metabolism with rat and human subjects [12]-[15]. The principal products are 6-hydroxymelatonin (Figure 1) and N-acetylseratonin (Figure 2) 
from O-demethylation. Hydroxylation occurred to a greater extent [12]. Both reactions have extensive precedent. These findings provide a base for application of the unifying theme of ET-ROS-OS. O-Demethylation of the hydroxylated metabolite should yield a catechol (Figure 3) which is commonly oxidized to an ET o-quinone (Figure 4). N-Acetylserotonin might conceivably be oxidized to the ET iminoquinone (Figure 5). Analogy for Figure 5 can be found from metabolism of psilocybin [16].

Representative examples are provided from the literature dealing with AO properties of melatonin [17]-[21].<smiles>NC(Cc1c[nH]c2ccccc12)C(=O)O</smiles><smiles>Cn1cc(CC(N)C(=O)O)c2cc(O)ccc21</smiles><smiles>CCCc1c[nH]c2ccc(O)cc12</smiles>

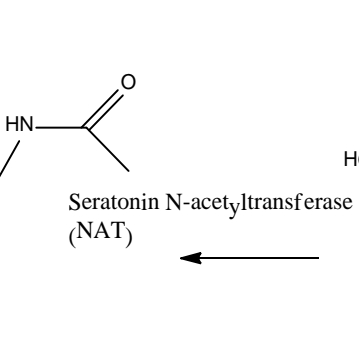

N-acetylseratonin<smiles>NCCc1c[nH]c2ccc(O)cc12</smiles>

Seratonin<smiles>COc1ccc2[nH]cc(CCNC(C)=O)c2c1</smiles>

Melatonin

Scheme 1. Biosynthesis of melatonin from tryptophan.<smiles>COc1cc2c(CCNC(C)=O)c[nH]c2cc1O</smiles>

Figure 1. 6-hydroxymelatonin.<smiles>CC(=O)NCCc1c[nH]c2ccc(O)cc12</smiles>

Figure 2. N-acetylserotonin. 

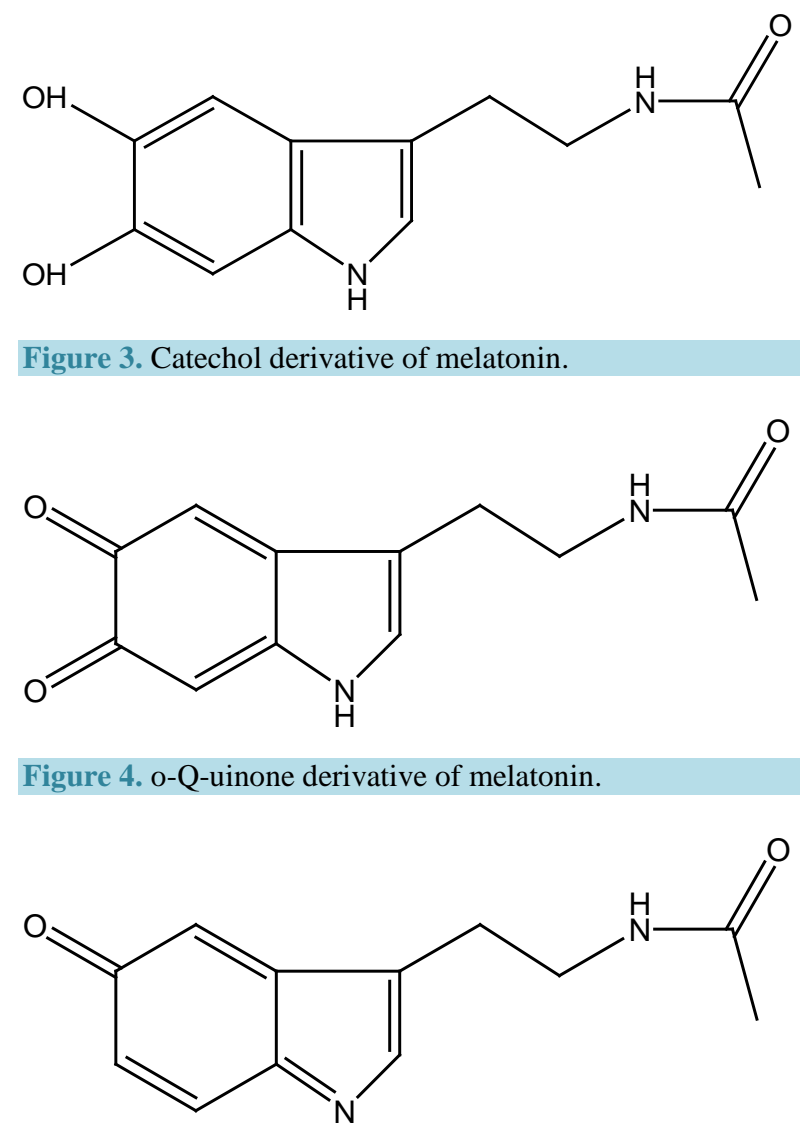

Figure 5. Iminoquinone derivative of N-acetylserotonin.

In relation to mechanism, a suggestion involves the indole nitrogen, presumably acting via the indole NH groupin terminating ROS [22]. Another possibility entails participation of the N-acetylseratonin metabolite. Phenols comprise a well-known class of AOs, e.g., vitamin E, which operate by way of electron or hydrogen atom donation [23]. Electrical effects, which are common in the brain, might be a result of participation, in part, of the postulated ET iminoquinone and o-quinone.

\section{Cancer}

Over the decades, many factors have been found to be involved in the biochemistry of cancer, including electron transfer, reactive oxygen species and oxidative stress [24]-[26], which are addressed in other articles. This section provides a general approach involving many factors linking circadian rhythm and cancer.

Circadian rhythms show universally a 24-hour oscillation pattern, in metabolic physiological and behavioral functions of almost all species, due to a fundamental adaptation to rotation of earth around its own axis [27]. Molecular mechanisms of generation of circadian rhythms organize a biochemical network in suprachiasmatic nucleus and peripheral tissues, building cell autonomous clock pacemakers. Rhythmicity is observed in transcriptional expression of a wide range of clock-controlled genes which regulate a variety of normal cell functions like cell division and proliferation. Desynchrony of this rhythmicity seems to be implicated in several pathologic conditions, including tumorigenesis and progression of cancer. In 2007, the International Agency for Research in cancer categorized shift work that involves circadian desynchrony, as being probably carcinogenic to humans. A review discusses the potential relation between the disruption of normal circadian rhythms with the genetic driving machinery of cancer. Elucidation of the role of clockwork disruption, like the exposure to light-at-night and sleep disruption, in cancer biology, could be important in the development of new targeted anticancer therapies, in optimization of individualized chronotherapy and in modification of lighting environment in working environments. 
Circadian rhythms are commonly disrupted in women undergoing chemotherapy for breast cancer [28]. Bright light improves and strengthens circadian rhythms. A randomized controlled study examined the effect of morning administration of bright light therapy on circadian rhythms in women undergoing chemotherapy for breast cancer [28]. It was hypothesized that women receiving bright light therapy would exhibit more robust rhythms than women exposed to dim light. The results suggest that morning administration of bright light may protect women from experiencing circadian rhythm deterioration during chemotherapy.

Breast cancer is the worldwide leading cause of cancer incidence among women [29]. Night shift work exposure has been recently considered one of the significant breast cancer risk factors in industrialized countries. The mechanisms by which this works exposure may be responsible for cancer development is still discussed. In the last 15 years, many authors have paid attention to the relationship between night shift work and breast cancer risk. Both the circadian system alteration and the melatonin output reduction, related to the exposure to light-at-night during night shift work, remain the most valid hypotheses on the casual relation of shift work and breast cancer. Overall, the results suggest that there is an association between night shift work and breast cancer development in western countries. However, further studies are needed to confirm such association and to understand which biomolecular mechanisms may be involved in the pathogenesis of cancer diagnosed in patients with night shift work exposure.

Disruption of the circadian rhythm has been hypothesized to increase cancer risk, either because of direct disruption of the molecular machinery generating circadian rhythms or because of disruption of parameters controlled by the clock, such as melatonin levels or sleep duration [30]. Data are sparse about potential effects of circadian disruption on the risk of prostate cancer. A review systematically examines available data evaluating the effects of light at night, sleep patterns, and night shift work on prostate cancer risk.

Circadian clocks are endogenous molecular time-keeping systems that underlie daily fluctuations in multiple physiological and biochemical processes [31]. It is now well recognized that dysfunction of the circadian system may be associated with a heightened incidence of cancer. A review presents evidence supporting the important role played by the circadian clock in the development of cancer and the therapeutic efficacy of anticancer agents. A number of circadian clock genes have been identified. Continued research should increase understanding of the role of circadian clocks which could, ultimately, reduce the incidence of cancer in people with disrupted sleep-wake cycles, such as shift workers and flight attendants, and provide optimal chronopharmacology for cancer treatment.

Hormone secretion, metabolism, and the cell cycles are under rhythmic control [32]. Lack of rhythmic control has been predicted to lead to uncontrolled proliferation and cancer. Consistent with the prediction are findings that circadian disruption by dim light at night or chronic jet lag accelerates tumor growth in desynchronized animals. Circadian controlled factors have been implicated in controlling tumor growth in these animals. Recent attention has focused on the signaling pathways activated by the circadian controlled factors because these pathways hold potential for the development of novel strategies for cancer prevention and treatment.

An investigation involves comparison of circadian characteristics for cytotoxic lymphocytes in cell lung cancer patients versus controls [33]. Timed circadian administration (chronotherapy) in immunotherapy and other cancer treatments may improve efficacy due to persistent circadian entrainment of healthy tissues.

A review article discusses recent work on the melatonin-mediated circadian regulation and integration of molecular, dietary, and metabolic signaling mechanisms involved in human breast cancer growth and the consequences of circadian disruption by exposure to light at night [34]. Experimental evidence in rats and humans indicates that light at night-induced circadian disruption of the nocturnal melatonin signal activates human breast cancer growth, metabolism, and signaling which provides the strongest mechanistic support for population and ecological studies demonstrating elevated breast cancer risk in night shift workers and other individuals increasingly exposed to light at night.

Little is known about the relationship between sleep parameters and fatigue in patients at the initiation of radiation therapy [35]. A significant percentage of oncology patients experience significant disturbances in sleep-wake circadian activity rhythms at the initiation of radiation therapy. The disturbances occur in both sleep initiation and sleep maintenance. Epidemiologic studies show high incidence of cancer in shift workers [36]. The finding suggests that circadian disruption induces the progression of malignant tumors via a Wnt signaling pathway.

Cell proliferation in all rapidly renewing mammalian tissues follows a circadian rhythm that is often disrupted in advanced-stage tumors [37]. Epidemiologic studies have revealed a clear link between disruption of circadian 
rhythms and cancer development in humans. Mice lacking certain circadian genes are deficient in cell cycle regulation and Per2 mutant mice are cancer-prone. However, it remains unclear how circadian rhythm in cell proliferation is generated in vivo and why disruption of circadian rhythm may lead to tumorigenesis. Tumor suppression in vivo is a clock-controlled physiological function. The central circadian clock paces extracellular mitogenic signals that drive peripheral clock-controlled expression of key cell cycle and tumor suppressor genes to generate a circadian rhythm in cell proliferation. Frequent disruption of circadian rhythm is an important tumor promoting factor.

Prostate cancer is a major age-related malignancy as increasing age correlates with increased risk for developing this neoplasm [38]. Similarly, alterations in circadian rhythms have also been associated with the aging population and cancer risk. The pineal hormone melatonin is known to regulate circadian rhythms, which is under the control of a core set of genes. The data suggest that melatonin should be thoroughly investigated as an agent for the management of prostate cancers and other age-related malignancies.

Circadian disruption accelerates cancer progression, whereas circadian reinforcement could halt it [39]. Mice with pancreatic adenocarcinoma were synchronized and fed ad libitum or with meal timing. The reinforcement of the host circadian timing system with meal timing translated into cancer growth inhibition. Targeting circadian clocks represents a novel potential challenge for cancer therapeutics.

Breast cancer incidence increases rapidly as societies industrialize. Many changes occur during the industrialization process, one of which is dramatic alteration in the lighted environment from a sun-based system to an electricity-based system [40]. Increasingly, the natural dark period at night is being seriously eroded for the bulk of humanity. Based on the fact that light during the night can suppress melatonin, and also disrupt the circadian rhythm, it was proposed in 1987 that increasing use of electricity to light the night accounts in part for the rising risk of breast cancer globally. Predictions from the theory include: non-day shift work increases risk, blindness lowers risk, long sleep duration lowers risk, and population level community nighttime light level co-distributes with breast cancer incidence. Thus far, studies of these predictions are consistent in support of the theory. A new avenue of research has been on function of circadian genes and whether these are related to breast cancer risk.

The circadian clock is an endogenous time keeping system shared by most organisms [41]. In mammals, a master pacemaker in the hypothalamus orchestrates temporal alignment of behavior and physiology by transmitting daily signals to multiple clocks in peripheral tissues. Disruption of this communication has a profound effect on human health and has been linked to diverse pathogenic conditions, including cancer. At the center of the molecular circadian machinery is a set of clock genes generating rhythmic oscillations on a cellular level. In the past several years, research from different fields has revealed the complexity and ubiquitous nature of circadian regulation, uncovering intriguing associations between clock components and cellular pathways implicated in tumorigenesis. A review discusses the emerging role of circadian genes in hematological and hormone-related malignancies. New insights suggest that manipulating circadian biology as a way to fight cancer, as well as other life threatening diseases, is within the realm of possibility.

Some reports have indicated that the core clock gene, PER2, regulates the cell cycle, immune systems and neural functions (Scheme 2) [42]. To understand the effects of PER2 on tumor growth in vivo, stable transformants of murine sarcoma cell line expressing different levels of PER2 were established. The growth of stable PER2 transformants in vivo was significantly and dose-dependently suppressed according to the amount of PER2 expressed, indicating that PERs plays a role in the growth suppression of sarcoma cells. The results suggest that sarcoma cells overexpressing PER2 suppress tumors in vivo by changing the nature of tumor cell adhesion.

The circadian timing is composed of molecular clocks, which drive 24 hour changes in xenobiotic metabolism and detoxification, cell cycle events, DNA repair, apoptosis, and angiogenesis [43]. The cellular circadian clocks are coordinated by endogenous physiological rhythms, so that they tick in synchrony in the host tissues that can be damaged by anticancer agents. As a result, circadian timing can modify the tolerability of anticancer medications. Improved efficacy is also seen when drugs are given near their respective times of best tolerability, due to a) inherently poor circadian entrainment of tumors and b) persistent circadian entrainment of healthy tissues. Conversely, host clocks are disrupted whenever anticancer drugs are administered at their most toxic time. On the other hand, circadian disruption accelerates experimental and clinical cancer processes. Gender, circadian physiology, clock genes, and cell cycle critically affect outcome on cancer chronotherapeutics.

A disruption of the circadian timing system, as identified by monitoring maker biorhythms, is common in 


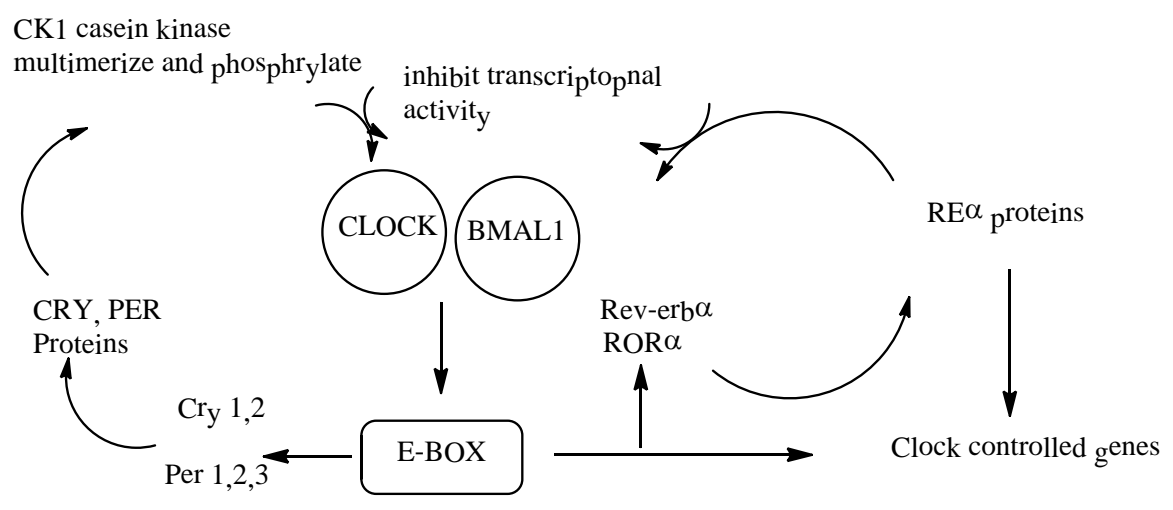

Scheme 2. A schematic model of molecular clockwork of the circadian clock in mammals.

cancer patients [44]. A body of evidence paves the path for innovative therapeutic approaches targeting the circadian timing system in an effort to diminish constitutional symptoms induced by cancer and some anticancer treatments.

Practical circadian therapy for the cancer patient involves 3 spheres of intervention, namely improving lifestyle, optimizing internal biochemical milieu, and adjusting treatment times [45]. The potential value of improving overall circadian functioning is shown in the work in which pronounced rest-activity rhythms were associated with better survival in colorectal cancer patients receiving chronomodulated chemotherapy. Lifestyle interventions that may improve circadian functioning involve diet, physical activity, and mind-body therapies. A diet that is ant-inflammatory and has appropriate carbohydrate intake, as well as regular meal timing, encourages normal circadian cycles. Adequate daytime physical activity encourages restful sleep, and morning light exposure during exercise may entrain melatonin rhythms. Meditation and other mind-body therapies can reduce anxiety and depression that may disrupt sleep. Aspects of the biochemical milieu that specifically disrupt circadian functioning are inflammation and stress hormones. Inflammation and cytokine disruption can be addressed with diet, herbs, and other natural substances. Chronomodulation of chemotherapy is discussed. Integrating all the above treatment modalities has the potential to improve both the quality of life and disease outcomes in cancer patients.

In industrialized countries, certain types of cancer, most notably breast and prostate, are more frequent than in poorly developed nations [46]. This high cancer frequency is not explained by any of the conventional causes. Within the past decade, numerous reports have appeared that link light at night with an elevated cancer risk. The three major consequences of light-mediated perturbation of circadian timing are sleep deprivation, chronodisruption, and melatonin suppression. Each of these individually or in combination may contribute to the reported rise in certain types of cancer. In the article, the potential mechanisms underlying the basis of the elevated cancer risk are briefly discussed. Finally, if cancer is a consequence of excessive nighttime light, it is likely that other diseases/conditions may also be exaggerated by the widespread use of light after darkness onset.

The circadian timing system coordinated by the suprachiasmatic nuclei (SCN) of the hypothalamus regulates daily rhythms of behavior, physiology, as well as cellular metabolism and proliferation [47]. Altered circadian rhythms predict for poor survival of cancer patients. An increased incidence of several cancers has been reported in flight attendants and in shift workers. The results support the role of circadian timing system in cancer progression and call for the development of therapeutic strategies aimed at preventing or treating circadian clock dysfunction.

Sirtuins appear to play a critical role in the process of carcinogenesis, especially in age-related neoplasm [48]. Similarly, alterations in circadian rhythms, as well as production of the pineal hormone melatonin, have been linked to aging and cancer risk. Melatonin has been found to act as a differentiating agent in some cancer cells and to lower their invasive and metastatic status. In addition, melatonin synthesis and release occur in a circadian rhythm fashion which has been linked to the core circadian machinery genes. Recent studies have linked sirtuins to the circadian rhythm machinery through direct deacetylation activity, as well as through other means. In this review, evidence is provided for a possible connection between sirtuins, melatonin, and the circadian rhythm circuitry and their implications in aging, chronomodulation, and cancer.

Circadian rhythms govern a remarkable variety of metabolic and physiological functions [49]. Accumulating 
evidence indicates that the disruption of circadian rhythms might be directly linked to cancer. Several molecular gears constituting the clock machinery have been found to establish functional interplays with regulators of the cell cycle, and alterations in clock function could lead to aberrant cellular proliferation. Connections between the circadian clock and cellular metabolism have been identified that are regulated by chromatin remodeling. This suggests that abnormal metabolism in cancer could also be a consequence of a disrupted circadian clock. Therefore, a comprehensive understanding of the molecular links that connect the circadian clock to the cell cycle and metabolism could provide therapeutic benefit against certain human neoplasm.

The first whole genome inspections of mutations in human colon and breast cancer have observed specific retained clock gene mutations [50]. Single nucleotide polymorphism within the gene of clock, clock-controlled, and melatonin pathways have been found to confer excess cancer risk or protection from cancer. Experimental studies have shown specific core clock genes (PER2 and PER1) are tumor suppressors because their genetic absence doubles tumor numbers; and decreasing their expression in cancer cells doubles cancer growth rate, whereas their overexpression decreases cancer growth rate and diminishes tumor numbers. Experimental interference with circadian clock function increases cancer growth rate, and clinical circadian disruption is associated with higher cancer incidence, faster cancer progression, and shorter cancer patient survival. Patients with advanced lung cancer suffering greater circadian activity/sleep cycle disruption undergo greater interference with function, greater anxiety and depression, poorer nighttime sleep, greater daytime fatigue, and poorer quality of life than comparable patients who maintain good circadian integration. Screening for small molecules in targeting the circadian clock to stabilize its phase and enhance its turn is likely to help prevent and control human cancer. Cellular proliferation and the expression of cell cycle regulators are also controlled by the circadian clock [51]. The data suggest PER1 has tumor-suppressor function that diminishes cancer proliferation and tumor growth, but only at specific times of day.

A study examined patterns of circadian activity rhythms and their relationship with fatigue. Anxiety/depression, and demographic/medical variable were investigated in women receiving breast cancer adjuvant therapy treatments [52]. Disruption patterns of circadian activity rhythms were prevalent and associated with distressing fatigue and depressive symptoms during chemotherapy and at recovery. The intervention resulted in more robust rhythms. The possibility of a portion of the breast cancer burden might be explained by the introduction and increasing use of electricity to light the night was suggested $>20$ years ago [53]. The theory is based on nocturnal light-induced disruption of circadian rhythms, notably reduction of melatonin synthesis. The basic understanding of phototransduction for the circadian system and of the molecular genetics of circadian rhythm generation are both advancing rapidly, and will provide for the development of lighting technologies at home and work that minimizes circadian disruption, while maintaining visual efficiency and aesthetics.

A report concludes that the expression pattern of circadian genes might be a biomarker for the prognosis of breast cancer [54]. A study dealt with modulation of circadian rhythm of DNA synthesis in tumor cells by inhibiting platelet-derived growth factor signaling [55]. PER2, a core circadian clock gene, has tumor suppressor properties and is mutated or down regulated in human breast cancers [56]. The expression of this gene was manuplated in vitro and in vivo to more fully understand how the PER2 clock gene product affects growth. PER2 and perhaps other clock genes represent a class of potential therapeutic targets whose manipulation will modulate cancer growth and cancer cell proliferation.

The circadian timing system controls drug metabolism and cellular proliferation over the 24 hour day through molecular clocks in each cell [57]. These cellular clocks are coordinated by a hypothalamic pacemaker, the suprachiasmetic nuclei, that generate or controls circadian physiology. The circadian timing system plays a role in cancer processes and their treatments through the downregulation of malignant growth and the generation of large and predictable 24 hour changes in toxicity and efficacy of anti-cancer drugs. The tight interactions between circadian clocks, cell division cycle and pharmacology pathways have supported sinusoidal circadian-based delivery of cancer treatments. Such chronotherapeutics have been mostly implemented in patients with metastatic colorectal cancer, the second most common cause of death from cancer. Stochastic and deterministic models of the interactions between circadian clock, cell cycle and pharmacology confirmed the poor therapeutic value of both constant-rate and wrongly timed chronomodulated infusions.

The circadian clock cell cycle interacts at the level of genes, proteins, and biochemical signals [58]. The disruption or reinforcement of the host circadian timing system accelerates or slows down cancer growth through modifications of host and tumor circadian clocks. Thus, cancer cells not only display mutation of cell cycle genes, but also exhibit severe defects in clock gene expression levels or 24 hour patterns, which can in turn favor 
abnormal proliferation. Mathematical models show that the therapeutic index of chemotherapeutic drugs can be optimized through distinct delivery profiles, depending on the initial host/tumor status and variability in circadian entrainment and/or cell cycle length.

Proper circadian regulation is essential for the well being of the organism, and disruption of circadian rhythms is associated with pathological conditions including cancer [59]. In mammals, the core clock genes, PER1 and PER2, are key regulators of circadian rhythms, both in the central clock, in the hypothalamus and in peripheral tissues (Scheme 2). Findings revealed molecular links between PER genes and cellular components that control fundamental cellular processes, such as cell division and DNA damage. Data shed light on mechanisms by which circadian oscillators operate in peripheral organs to influence tissue-dependent metabolic and hormonal pathways. Circadian cycles are linked to basic cellular expression and protein interactions. By controlling global networks, such as chromatin remodeling and protein families, which themselves regulate a broad range of cellular functions, circadian regulation impinges upon almost all major physiological functions.

An investigation examined effects of exogenous melatonin and circadian synchronization on tumor progression in mice [60]. The circadian organization is responsible for predictable changes in the tolerability and efficacy of anticancer agents, and also controls tumor promotion and growth [61]. The clinical relevance of the chronotherapy principle, i.e., treatment administration as a function of rhythms, has been demonstrated in randomized multicenter trials, using programmable-in-time drug delivery systems. Chronotherapeutic schedules first documented the safety and the activity of oxaliplatin-based combination chemotherapy in patients with metastatic colorectal cancer. The chronotherapy concept offers further promise for improving current cancer treatment options, as well as for optimizing the development of new anticancer agents.

A meeting reported the role of environmental lighting and circadian disruption in cancer and other diseases [62]. Overexpression of either PER1 or PER2 in cancer cells inhibits their neoplastic growth and increases their apoptotic rate [63]. In vivo studies showed that mice deficient in PER2 showed significantly higher incidences of tumor development after genotoxic stress. Loss and dysregulation of PER1 and PER2 gene expression have been found in many types of human cancers. Studies demonstrate that both PER1 and PER2 are involved in damage response pathways and implicate normal circadian function as a factor in tumor suppression. The molecular pathways that may couple the circadian machinery to breast cancer are elaborated [64].

\section{Aging}

The causes of aging and their treatment and prevention have attracted much scientific attention. One theory of aging which has achieved increasing acceptance is the free radical theory of aging [1]. Our review presents representative examples from the extensive literature dealing with the involvement of circadian rhythm with aging. It is well established that increasing age is associated with increased cancer risk.

Aging affects all organic structures and processes, including the circadian system and its principal sign, the biological rhythms [65]. Aging produces losses in function of three components: receptors (the eye), the central pacemaker and the outputs. This leads to the alteration of overt rhythms, with losses in the phase relationship between them.

Circadian rhythms are generally determined biological rhythms that are considered an important adaptive mechanism to the cyclical light/dark alterations in the earth environment [66]. Age-related changes in the circadian time-keeping mechanism are well known, and seemingly contribute to various pathologies of aging. The circadian system and circadian proteins play direct roles in many physiological processes, including those associated with aging. Core circadian proteins, in addition to their known functions in the circadian oscillator, play an essential non-redundant role in the control of tissue homeostasis and aging. The involvement of circadian proteins in the regulation of metabolism, genotoxic stress response and ROS homeostasis can be responsible for the premature aging observed in some circadian mutants.

Aging alters numerous aspects of circadian biology, including the amplitude generated by the suprachiasmatic nuclei (SCN) of the hypothalamus, the site of the central circadian pacemaker in mammals, and the response of the pacemaker to environmental stimuli, such as light [67]. Age alters the $24 \mathrm{~h}$ expression profile of the clock and its binding partner Bmal1 genes in the hamster SCN.

Effects of aging on the circadian rhythm of locomotor activity in males of Dorsophila nasuta were investigated [68]. Aging is associated with weakened coupling between the activity-rest cycle and the circadian clock [69]. 
The relationship between aging and daily circadian behavior in humans is bidirectional: on the one hand, dysfunction of circadian clocks promotes age-related maladies; on the other, aging per se leads to changes and disruption in circadian behavior and physiology [70]. For the latter case, recent research suggests that changes to both homeostatic and circadian sleep regulatory mechanisms may play a role.

A review discusses the ways in which the circadian rhythms of older people are different from those of younger adults [71]. Older people undoubtedly have earlier circadian phases than younger adults, and have more trouble coping with shift work and jet lag.

Chronic disruptions in circadian function are associated with an increase in a variety of disease states, including heart disease, ulcers and diabetes [72]. With advanced age, the genes regulating circadian function at the cellular level become disorganized. The review explores aging-associated changes in circadian function and examines evidence linking such alterations to adverse health consequences in late life and promotion of the aging process.

Aging in zebrafish is associated with major but selective circadian alterations [73]. By 3-5 years of age, zebrafish have reduced amplitude and increased fragmentation of entrained circadian rhythms of activity, with fast desynchronization of the rhythms. Aging is also associated with a reduction in the overall duration of nighttime sleep, followed by a higher arousal threshold during day. All 24-hour endocrine rhythms partially reflect the interaction of circadian rhythmicity with sleep-wake homeostasis [74]. The decrease in sleep quality which characterizes aging may contribute to age-related alterations in hormonal function and their metabolic consequences. Literature is reviewed indicating that aging is characterized by changes in circadian rhythms and sleep quality [75]. The most marked change is an attenuation of amplitude. An advance of phase, a shortening of period, and a desynchronization of rhythms are also evident. Circadian disruption induced by light-at-night accelerates aging and promotes tumorigenesis in rats [76] [77]. Shift-work that involves circadian disruption is probably carcinogenic to humans.

Circadian clock gene expression is changed in human pathologies, and transgenic mice with mutations in clock genes develop cancer and premature aging [78]. There is circadian clock-dependent control of cell proliferation, together with control of metabolism, oxidative and genotoxic stress response, and DNA repair.

A review is dedicated to the age-related changes in circadian rhythms as they occur in humans [79]. Alterations in the regulation of circadian rhythms are thought to contribute to the symptoms of a number of conditions for which the risk is increased in old age (e.g., sleep disturbances, dementia, and depression). During aging, and in particular in Alzheimer's disease, circadian rhythms are disturbed [80]. These disturbances include phase advance, reduced period and amplitude, increased intradaily variability and a decreased interdaily stability of the rhythm. Among the factors underlying these changes, the loss of SCN neurons seems to play a central role.

In human beings and animal models, cognitive performance is often impaired in natural and experimental situations where circadian rhythms are disrupted [81]. This includes a general decline in cognitive ability and fragmentation of behavioral rhythms in the aging population of numerous species. There is evidence that rhythm disruption may lead directly to cognitive impairment. These results support the notion that age-related rhythm fragmentation contributes to the age-related memory decline.

Resetting the circadian clock leads to well being and increased life span, whereas clock disrupting is associated with aging and morbidity [82]. Increased longevity and improved health can be achieved by different feeding regimens that reset circadian rhythms and may lead to better synchrony in metabolism and physiology. A review focuses on the relationship between circadian rhythms, aging attenuation, and life-span extension in mammals. An article deals with circadian sleep, illumination, and activity patterns involving influences of aging and time reference [83].

Sleep/wake and circadian rest-activity rhythms become irregular with age [84]. Typical outcomes include fragmented sleep during the night, advanced sleep phase syndrome and increased daytime sleepiness. These changes lead to a reduction in the quality of life due to cognitive impairments and emotional stress. More importantly, severely disrupted sleep and circadian rhythms have been associated with an increase in disease susceptibility. Additionally, many of the same brain areas affected by neurodegenerative diseases include the sleep and wake promoting systems. An article discusses the relationship between circadian clock gene, circadian rhythmicity, aging-related phenotypes, and cancer [85] [86].

Circadian rhythm disturbances, such as sleep disorders, are frequently seen in aging and are even more pronounced in Alzheimer's disease (AD) [87]. Alterations in the biological clock, the suprachiasmatic nucleus (SCN), and the pineal gland during aging and $\mathrm{AD}$ are considered to be the biological basis for these circadian 
rhythm disturbances. Pineal melatonin secretion and pineal clock gene oscillation were disrupted in AD patients. Circadian rhythms have are disturbed as a consequence of both normal aging and age-associated pathologies, like Alzheimer's disease (AD) [88]. Because sleep loss in young subjects results in endocrine disturbances which mimic those observed in aging, it is conceivable that the decrease in sleep quality which characterizes aging may contribute to age-related alterations in hormonal function and their metabolic consequences [89].

Chronic, night administration of melatonin to aging mice and transplantation of a young pineal gland into the rhythmic rudiment of older mice and rats have been studied with the aim of evaluating their effects on aging of gonadal, sexual, and reproductive functions. Both melatonin administration and young-to-old pineal grafting positively affected size and function of testes and maintenance of juvenile hippocampal and testicular LHRH-receptors and beta-adrenergic receptors in the testes of old rats and mice. Results demonstrate that a pineal-directed circadian function and cyclicity is fundamental for the regulation of sexual reproductive physiology, and that proper intervention with melatonin may potentially postpone aging effects of both neural and gonadal sexual function [90].

Data indicate that there are dramatic changes in light-activated molecular responses in the suprachiasmatic nuclei of old hamsters, and suggest that these molecular changes may underlie age-related changes in the effects of light on the circadian clock system [91].

\section{Conclusion}

Melatonin, a pineal hormone, plays an important role in operations of the circadian clock. The focus is on influences surrounding cancer and aging. The mechanism based on ET-ROS-OS appears to occupy a fundamental role. The multifaceted approach puts focus on melatonin and circadian rhythm in relation to various physiological processes.

\section{Acknowledgements}

Editorial assistance by Linda Muroi, Bianca Aviña and Thelma Chavez is acknowledged, in addition to valuable contributions by Kathleen McNamara.

\section{References}

[1] Harman, D. (2009) Origin and Evolution of the Free Radical Theory of Aging: A Brief Personal History. Biogerontology, 10, 773-781. http://dx.doi.org/10.1007/s10522-009-9234-2

[2] Everts, S. (2012) Picking Apart Our Circadian Clock. Chemical Engineering News, 90, 30-32.

[3] Borijigin, J., Li, X. and Snyder, S.H. (1999) The Pineal Gland and Melatonin: Molecular and Pharmacologic Regulation. Annual Review of Pharmacology and Toxicology, 39, 53-65. http://dx.doi.org/10.1146/annurev.pharmtox.39.1.53

[4] Simonneaux, V. and Ribelayga, C. (2003) Generation of the Melatonin Endocrine Message in Mammals: A Review of the Complex Regulation of Melatonin Synthesis by Norepinephrine, Peptides, and Other Pineal Transmitters. Pharmacological Reviews, 55, 325-395. http://dx.doi.org/10.1124/pr.55.2.2

[5] Chowdhury, I., Sengupta, A. and Maitra, S.K. (2008) Melatonin: Fifty Years of Scientific Journey from the Discovery in Bovine Pineal Gland to Delineation of Functions in Human. Indian Journal of Biochemistry Biophysics, 45, 289304.

[6] Liu, C., Weaver, D.R., Jin, X., Shearman, L.P., Pieschl, R.L., Gribkoff, V.K. and Reppert, S.M. (1997) Molecular Dissection of Two Distinct Actions of Melatonin on the Suprachiasmatic Circadian Clock. Neuron, 19, 91-102. http://dx.doi.org/10.1016/S0896-6273(00)80350-5

[7] Liu, C., Weaver, D.R. and Reppert, S.M. (1997) Cellular Construction of a Circadian Clock: Period Determination in the Suprachiasmatic Nuclei. Cell, 91, 855-860. http://dx.doi.org/10.1016/S0092-8674(00)80473-0

[8] Hatter, S., Lucas, R.J., Mrosovsky, N., Thompson, S., Douglas, R.H., Hankins, M.W., Lem, J., Biel, M., Hofman, F., Foster, R. and Yau, K.W. (2003) Melanopsin and Rod Cone Photoreceptive Systems Account for All the Major Accessory Visual Functions in Mice. Nature, 424, 76-81.

[9] Reiter, R.J., Tan, D.X., Sanchez-Barcelo, E. and Mediavilla, M.D. (2011) Circadian Mechanism in the Regulation of Melatonin Synthesis: Disruption with Light at Night and the Pathophysiological Consequences. Journal of experimental and Integrative Medicine, 1, 13-22.

[10] 1Kovacic, P. and Somanathan, R. (2013) Multifaceted Approach to Circadian Rhythm: Redox, Oxidative Stress, Melatonin, Antioxidants, Nitric Oxide, Hypoxia, Anesthetics, Cortisol and Cocaine. Current Opinion in Chemical Biology, 
in Press.

[11] Rivera, S., Mor, M., Bedini, A., Spardone, G. and Tarzia, G. (2008) Melatonin Receptor Agonist: SAR and Application to the Treatment of Sleep-Wake Disorders. Current Topics in Medicinal Chemistry, 8, 954-968. http://dx.doi.org/10.2174/156802608784936719

[12] Young, I.M. (1985) Melatonin Is Metabolized to N-Acetyl Serotonin and 6-Hydroxymelatonin in Man. The Journal of Clinical Endocrinology Metabolism, 60, 114-119. http://dx.doi.org/10.1210/jcem-60-1-114

[13] Leone, R.M. and Silman, R.E. (1984) Melatonin Can be Differentially Metabolized in Rat to Produce N-Acetylseratonin in Addition to Hydroxymelatonin. Endocrinology, 114, 1825-1832. http://dx.doi.org/10.1210/endo-114-5-1825

[14] Leone, A.M., Francis, P.L. and Silman, R.E. (1987) The Isolation, Purification and Characterization of the Principal Urinary Metabolites of Melatonin. Journal of Pineal Research, 4, 253-266. http://dx.doi.org/10.1111/j.1600-079X.1987.tb00863.x

[15] Semak, I., Korik, E., Antonova, M., Wortsman, J. and Slominski, A. (2008) Metabolism of Melatonin by Chytochrome P-450 s in Rat Liver Mitochondria and Microsomes. Journal of Pineal Research, 45, 515-523. http://dx.doi.org/10.1111/j.1600-079X.2008.00630.x

[16] Kovacic, P. and Somanathan, R. (2009) Novel, Unifying Mechanism for Mescaline in the Centrak Nervous System. Oxidative Medicine and Cellular Longevity, 2, 1-10.

[17] Bai, J., Dong, L., Song, Z., Ge, H., Cai, X., Wang, G. and Liu, P. (2013) The Role of Melatonin as an Antioxidant in Human Lens Epithelial Cells. Free Radical Research, 47, 635-642. http://dx.doi.org/10.3109/10715762.2013.808743

[18] Navarro-Alarcon, M., Ruiz-Ojeda, F.J., Blanca-Herrera, R.M. and Agil, A. (2013) Antioxidant Activity of Melatonin in Diabetes in Relation to the Regulkation and Levels of Plasma Cu, Zn, Fe, Mn, and Se in Zucker Diabetic Fatty Rats. Nutrition, 29, 785-789. http://dx.doi.org/10.1016/j.nut.2012.11.005

[19] Kara, A., Akman, S., Ozkanler, S., Tozoglu, U., Kalkan, Y., Canakci, C.F. and Tozoglu, S. (2013) Immune Modulatory and Antioxidant Effects of Melatonin in Experimental Peridontitis in Rats. Free Radical Biology Medicine, 55, 21-26. http://dx.doi.org/10.1016/j.freeradbiomed.2012.11.002

[20] Reiter, R.J., Tan, D.X., Rosales-Corral, S. and Manchester, L.C. (2013) The Universal Nature, Unequal Distribution and Antioxidant Functions of Melatonin and Its Derivatives. Mini Reviews in Medicinal Chemistry, 13, 373-384.

[21] Fagali, N. and Catalá, A. (2012) The Antioxidant Behavior of Melatonin and Structurak Analogues during Lipid Peroxidation Depends Not Only on Their Functional Groups But Also on the Assay System. Biochemical and Biophysical Research Communications, 423, 873-877. http://dx.doi.org/10.1016/j.bbrc.2012.06.060

[22] Halliwell, B. and Gutteridge, J.M.C. (2000) Free Radicals in Biology and Medicine. Oxford University Press, Oxford, 103-194.

[23] Kovacic, P. and Somanathan, R. (2006) Beneficial Effects of Antioxidants in Relation to Carcinogens, Toxins and Various Illnesses. In: Panglossi, H.V., Ed., Frontires in Antioxidants Research, NOVA, New York, Ch. 1, 1-38.

[24] Kovacic, P. and Jacintho, J.D. (2001) Mechanisms of Carcinogenesis: Focus on Oxidative Stress and Electron Transfer. Current Medicinal Chemistry, 8, 773-796. http://dx.doi.org/10.2174/0929867013373084

[25] Kovacic, P. and Somanathan, R. (2007) Mechanism of Tumorigenesis: Focus on Oxidative Stress, Electron Transfer and Antioxidants. In: Wong, D.K., Ed., Tumorigenesis Research Advances, Nova Biomedical Books, New York, Ch. 2, 23-66.

[26] Kovacic, P. and Somanathan, R. (2012) Cell Signaling and Cancer: Integrated, Fundamental Approach Involving Electron Transfer, Reactive Oxygen Species, and Antioxidants. In: Chatterjee, M. and Kashfi, K., Eds., Cell Signaling \& Molecular Targets in Cancer, Springer, New York, Ch. 12, 273-297. http://dx.doi.org/10.1007/978-1-4614-0730-0 12

[27] Savvidis, C. and Koutsilieris, M. (2012) Circadian Rhythm Disruption in Cancer Biology. Molecular Medicine, 18, 1249-1260.

[28] Neikrug, A.B., Rissling, M., Trofimenko, V., Liu, L., Natarajan, L., Lawton, S. and Parker, B.A. (2012) Ancoli-Israel, S. Bright Light Therapy Protects Women from Circadian Rhythm Desynchronization during Chemotherapy for Breast Cancer. Behavioral Sleep Medicine, 10, 202-216. http://dx.doi.org/10.1080/15402002.2011.634940

[29] Leonardi, G.C., Rapisarda, V., Marconi, A., Scalisi, A., Catalano, F., Proietti, L., Travali, S., Libra, M. and Fenga. C. (2012) Correlation of the Risk of Breast Cancer and Disruption of the Circadian Rhythm. Oncology Reports, 28, 418428.

[30] Sigurdardottir, L.G., Valdimarsdottir, U.A., Fall, K., Rider, J.R., Lockley, S.W., Schernhammer, E. and Mucci, L.A. (2012) Cancer Disruption, Sleep Loss, and Prostate Cancer Risk: A Systematic Review of Epidemiologic Studies. Cancer Epidemiology, Biomarkers Prevention, 21, 1002-1011. http://dx.doi.org/10.1158/1055-9965.EPI-12-0116

[31] Huang, X.L., Fu, C.J. and Bu, R.F. (2011) Role of Circadian Clocks in the Development and Therapeutics of Cancer. 
Journal of International Medical Research, 39, 2061-2066. http://dx.doi.org/10.1177/147323001103900601

[32] Greene, M.W. (2012) Circadian Rhythms and Tumor Growth. Cancer Letters, 318, 115-123. http://dx.doi.org/10.1016/j.canlet.2012.01.001

[33] Mazzoccoli, G., Sothern, R.B., Parrella, P., Muscarella, L.A., Razio, V.M., Giuliani, F., Polyakova, V. and Kvetnoy, I.M. (2012) Comparison of Circadian Characteristics for Cytotoxic Lymphocyte Subsets in Non-Small Cell Lung Cancer Patients versus Controls. Clinical and Experimental Medicine, 12, 181-194. http://dx.doi.org/10.1007/s10238-011-0153-6

[34] Blask, D.E., Hill, S.M., Dauchy, R.T., Xiang, S., Yuan, L., Duplessis, T., Mao, L., Dauchy, E. and Sauer, L.A. (2011) Circadian Regulation of Molecular, Dietary, and Metabolic Signaling Mechanisms of Human Breast Cancer Growth by the Nocturnal Melatonin Signal and the Consequences of Its Disruption by Light at Night. Journal of Pineal Research, 51, 259-269. http://dx.doi.org/10.1111/j.1600-079X.2011.00888.x

[35] Miaskowski, C., Lee, K., Dunn, L., Dodd, M., Aouzarat, B.E., West, C., Paul, S.M., Cooper, B., Wara, W. and Swift, P. (2011) Sleep-Wake Circadian Activity Rhythm Parameters and Fatigue in Oncology Patients before the Initiation of Radiation Therapy. Cancer Nursing, 34, 255-268. http://dx.doi.org/10.1097/NCC.0b013e3181f65d9b

[36] Yasuniwa, Y., Izumi, H., Wang, K.Y., Shimajiri, S., Sasaguri, Y., Kawai, K., Kasai, H., Shimada, T., Miyake, K., Kashiwagi, E., Hirano, G., Kidani, A., Akiyama, M., Han, B., Wu, Y., Ieiri, I., Higuchi, S. and Kohno, K. (2010) Circadian Disruption Accelerates Tumor Growth and Angio/Stromagenesis through a Wnt Signaling Pathway. PLoS ONE, 5, Article ID: e15330.

[37] Lee, S., Donehower, L.A., Herron, A.J., Moore, D.D. and Fu, L. (2010) Disrupting Circadian Homeostasis of Sympathetic Signaling Promotes Tumor Development in Mice. PLOS ONE, 5, Article ID: e10995. http://dx.doi.org/10.1371/journal.pone.0010995

[38] Jung-Hynes, B., Huang, W., Reiter, R.J. and Ahmad, N. (2010) Melatonin Resynchronizes Dysregulated Circadian Rhythm Circuitry in Human Prostate Cancer Cells. Journal of Pineal Research, 49, 60-68.

[39] Li, X.M., Delainay, F., Dulong, S., Claustrat, B., Zampera, S., Fujii, Y., Teboul, M., Beau, J. and Lévi, F. (2010) Cancer Inhibition through Circadian Reprogramming of Tumor Transcription with Meal Timing. Cancer Research, 70, 3351-3360. http://dx.doi.org/10.1158/0008-5472.CAN-09-4235

[40] Stevens, R.G., Blask, D.E., Brinard, G.C., Hansen, J., Lockley, S.W., Provencio, I., Rea, M.S. and Reinlib, L. (2007) The Role of Environmental Lighting and Circadian Disruption in Cancer and Other Diseases. Environmental Health Perspectives, 115, 1357-1362. http://dx.doi.org/10.1289/ehp.10200

[41] Gery, S. and Koeffler, H.P. (2007) The Role of Circadian Regulation in Cancer. Cold Spring Harbor Symposia on Quantitative Biology, 72, 459-464. http://dx.doi.org/10.1101/sqb.2007.72.004

[42] Miyazaki, K., Wakabayashi, M., Hara, Y. and Ishida, N. (2010) Tumor Growth Suppression in Vivo by Overexpression of the Circadian Component, PER2. Genes to Cells, 15, 351-358. http://dx.doi.org/10.1111/j.1365-2443.2010.01384.x

[43] Lévi, F., Pkyar, A., Dulong, S., Innamonato, P.F. and Clairambault, J. (2010) Circadian Timing in Cancer Treatments. Annual Review of Pharmacology and Toxicology, 50, 377-421. http://dx.doi.org/10.1146/annurev.pharmtox.48.113006.094626

[44] Innominato, P.F., Mormont, M.C., Rich, T.A., Waterhouse, J., Lévi, F.A. and Bjarnason, G.A. (2009) Circadian Disruption, Fatigue, and Anorexia Clustering in Advanced Cancer Patients: Implications for Innovative Therapeutic Approaches. Integrative Cancer Therapies, 8, 361-370. http://dx.doi.org/10.1177/1534735409355293

[45] Block, K.I., Block, P.B., Fox, S.R., Birris, J.S., Feng, A.Y., de la Torre, M., Nathan, D., Tothy, P., Maki, A.K. and Gyllenhaal, C. (2009) Making Circadian Cancer Therapy Practical. Integrative Cancer Therapies, 8, 371-386. http://dx.doi.org/10.1177/1534735409352028

[46] Reiter, R.J., Tan, D.X., Erren, T.C., Fuentes-Broto, L. and Paredes, S.D. (2009) Light-Mediated Perturbations of Circadian Timing and Cancer Risk: A Mechanistic Analysis. Integrative Cancer Therapies, 8, 354-360. http://dx.doi.org/10.1177/1534735409352026

[47] Filipski, E. and Lévi, F. (2009) Circadian Disruption in Experimental Cancer Processes. Integrative Cancer Therapies, 8, 298-302. http://dx.doi.org/10.1177/1534735409352085

[48] Jung-Haynes, B., Reiter, R.J. and Ahmad, N. (2010) Sirtuins, Melatonin and Circadian Rhythms: Building a Bridge between Aging and Cancer. Journal of Pineal Research, 48, 9-19. http://dx.doi.org/10.1111/j.1600-079X.2009.00729.x

[49] Sahar, S. and Sassone-Corsi, P. (2009) Metabolism and Cancer: The Circadian Clock Connection. Nature Reviews Cancer, 9, 886-896. http://dx.doi.org/10.1038/nrc2747

[50] Hrushesky, W.J., Grutsch, J., Wood, P., Yang, X., Oh, E.Y., Ansell, C., Kidder, S., Ferrans, C., Quiton, D.F., Reynolds, J., Du-Quiton, J., Levin, R., Lis, C. and Braun, D. (2009) Circadian Clock Manipulation for Cancer Prevention and Control and the Relief of Cancer Symptoms. Integrative Cancer Therapies, 8, 387-397. http://dx.doi.org/10.1177/1534735409352086 
[51] Yang, X., Wood, P.A., Ansell, C.M., Quiton, D.F., Oh, E.Y., Du-Quiton, J. and Hrushesky, W.J. (2009) The Circadian Clock Gene PER1 Suppresses Cancer Cell Proliferation and Tumor Growth at Specific Times of Day. Chronobiology International, 26, 1323-1339. http://dx.doi.org/10.3109/07420520903431301

[52] Berger, A.M., Wielgus, K., Hertzog, M., Fischer, P. and Farr, L. (2009) Patterns of Circadian Activity Rhythms and Their Relationship with Fatigue and Anxiety/Depression in Women Treated with Breast Cancer Adjuvant Chemotherapy. Supportive Care in Cancer, 18, 105-114. http://dx.doi.org/10.1007/s00520-009-0636-0

[53] Stevens, R.G. (2009) Light-at-Night, Circadian Disruption and Breast Cancer: Assessment of Existing Evidence. International Journal of Epidemiology, 38, 963-970. http://dx.doi.org/10.1093/ije/dyp178

[54] Kuo, S.J., Chen, S.T., Yeh, K.T., Hou, M.F., Chang, Y.S., Hsu, N.C. and Chang, J.G. (2009) Disturbance of Circadian Gene Expression in Breast Cancer. Virchows Archiv, 454, 467-474. http://dx.doi.org/10.1007/s00428-009-0761-7

[55] Nakagawa, H., Koyanagi, S., Kuramoto, Y., Yoshizumi, A., Matsunaga, N., Shimeno, H., Soeda, S. and Ohdo, S. (2008) Modulation of Circadian Rhythm of DNA Synthesis in Tumor Cells by Inhibiting Platelet-Derived Growth Factor Signaling. Journal of Pharmacological Sciences, 107, 401-407. http://dx.doi.org/10.1254/jphs.08080FP

[56] Yang, X., Wood, P.A., Oh, E.Y., Du-Quiton, J., Ansell, C.M. and Hrushesky, W.J. (2009) Down Regulation of Circadian Clock Gene Period2 Accelerates Breast Cancer Growth by Altering Its Daily Growth Rhythm. Breast Cancer Research and Treatment, 117, 423-431. http://dx.doi.org/10.1007/s10549-008-0133-z

[57] Lévi, F., Altinok, A., Clairambault, J. and Goldbeter, A. (2008) Implications of Circadian Clocks for the Rhythmic Delivery of Cancer Therapeutics. Philosophical Transactions of the Royal Society A: Mathematical, Physical and Engineering Sciences, 366, 3575-3598. http://dx.doi.org/10.1098/rsta.2008.0114

[58] Lévi, F., Filipski, E., Iurisci, I., Li, X.M. and Innominato, P. (2007) Cross-Talks between Circadian Timing System and Cell Division Cycle Determine Cancer Biology and Therapeutics. Cold Spring Harbor Symposia on Quantitative Biology, 72, 465-475. http://dx.doi.org/10.1101/sqb.2007.72.030

[59] Gery, S. and Koeffler, H.P. (2010) Circadian Rhythms and Cancer. Cell Cycle, 9, 1097-1103. http://dx.doi.org/10.4161/cc.9.6.11046

[60] Otálora, B.B., Madrid, J.A., Alvarez, N., Vicente, V. and Rol, M.A. (2008) Effects of Exogenous Melatonin and Circadian Synchronization on Tumor Progression in Melanoma-Bearing C57BL6 Mice. Journal of Pineal Research, 44, 307-315. http://dx.doi.org/10.1111/j.1600-079X.2007.00531.x

[61] Lévi, F. (2006) The Circadian Timing System, a Coordinator of Life Processes. Implications for the Rhythmic Delivery of Cancer Therapeutics. Proceedings of IEEE Engineering in Medicine and Biology Society, 6736-6739. http://dx.doi.org/10.1109/EMBS.2006.260934

[62] Stevens, R.G. (2009) Working against Our Endogenous Circadian Clock: Breast Cancer and Electric Lighting in the Modern World. Mutation Research, 680, 106-108. http://dx.doi.org/10.1016/j.mrgentox.2009.08.004

[63] Chen-Goodspeed, M. and Lee, C.C. (2007) Tumor Suppression and Circadian Function. Journal of Biological Rhythms, 22, 291-298. http://dx.doi.org/10.1177/0748730407303387

[64] Sahar, S. and Sassone-Corsi, P. (2007) Circadian Clock and Breast Cancer: A Molecular Link. Cell Cycle, 6, 13291331. http://dx.doi.org/10.4161/cc.6.11.4295

[65] Lucas-Sánchez, A., Martinez-Nicolas, A., Escames, G. and de Costa, J. (2011) Aging of the Circadian System. Revista Española de Geriatría y Gerontología, 47, 76-80. http://dx.doi.org/10.1016/j.regg.2011.09.008

[66] Kondratov, R.V. (2007) A Role of the Circadian System and Circadian Proteins in Aging. Ageing Research Reviews, 6, 12-27. http://dx.doi.org/10.1016/j.arr.2007.02.003

[67] Kolker, D.E., Fukuyama, H., Huang, D.S., Takahashi, J.S., Horton, T.H. and Turek, F.W. (2003) Aging Alters Circadian and Light-Induced Expression of Clock Genes in Golden Hamsters. Journal of Biological Rhythms, 18, 159-169. http://dx.doi.org/10.1177/0748730403251802

[68] Joshi, D., Barnabas, R., Martin, E.R., Parihar, V. and Kanojiya, M. (1999) Aging Alters Properties of the Circadian Pacemakers Controlling the Locomotor Activity Rhythm in Males of Drosophila Nasuta. Chronobiology International, 16, 751-758. http://dx.doi.org/10.3109/07420529909016942

[69] Van Reeth, O., Zhang, Y., Reddy, A., Zee, P. and Turek, F.W. (1993) Aging Alters the Entraining Effects of an Activity-Inducing Stimulus on the Circadian Clock. Brain Research, 607, 286-292. http://dx.doi.org/10.1016/0006-8993(93)91518-W

[70] Brown, S.A., Schmitt, K. and Eckert, A. (2011) Aging and Circadian Disruption: Causes and Effects. Aging, 3, 813817.

[71] Monk, T.H. (2005) Aging Human Circadian Rhythms: Conventional Wisdom May Not Always Be Right. Journal of Biological Rhythms, 20, 366-374. http://dx.doi.org/10.1177/0748730405277378

[72] Gibson, E.M., Williams, W.P. and Kriegsfeld, L.J. (2008) Aging in the Circadian System: Considerations for Health, 
Disease Prevention and Longevity. Experimental Gerontology, 44, 51-56. http://dx.doi.org/10.1016/j.exger.2008.05.007

[73] Zhdanova, I.V., Lopez-Patino, M., Shang, E., Kishi, S. and Guilin, E. (2008) Aging of the Circadian System in Zebrafish and the Effects of Melatonin on Sleep and Cognitive Performance. Brain Research Bulletin, 75, 433-441. http://dx.doi.org/10.1016/j.brainresbull.2007.10.053

[74] Froy, O. (2011) Circadian Rhythms, Aging, and Life Span in Mammals. Physiology, 26, 225-235. http://dx.doi.org/10.1152/physiol.00012.2011

[75] Myers, B.L. and Badia, P. (1995) Changes in Circadian Rhythms and Sleep Quality with Aging: Mechanism and Interventions. Neuroscience \& Biobehavioral Reviews, 19, 553-571. http://dx.doi.org/10.1016/0149-7634(95)00018-6

[76] Vinogradova, I.A., Anisimov, V.N., Bukalev, A.V., Semenchenko, A.V. and Zabezhinski, M.A. (2009) Circadian Disruption Induced by Light-at-Night Accelerates Aging and Promotes Tumorigenesis in Rats. Aging, 1, 855-865.

[77] Vinogradova, I.A., Anisimov, V.N., Bukalev, A.V., Semenchenko, A.V. and Zabezhinski, M.A. (2010) Circadian Disruption Induced by Light-at-Night Accelerates Aging and Promotes Tumorigenesis in Young, but Not in Old. Aging, 2, 82-92.

[78] Khapre, R.V., Samsa, W.E. and Kondratov, R.V. (2010) Circadian Regulation of Cell Cycle: Molecular Connections between Aging and the Circadian Clock. Annals of Medicine, 42, 404-415.

[79] Van Someren, E.J. (2000) Circadian Rhythms and Sleep in Human Aging. Chronobiology International, 17, $233-243$. http://dx.doi.org/10.1081/CBI-100101046

[80] Mirmiran, M., Swaab, D.F., Kok, J.H., Hofman, M.A., Witting, W. and Van Gool, W.A. (1992) Circadian Rhythms and the Suprachiasmatic Nucleus in Perinatal Development, Aging and Alzheimer's Disease. Progress in Brain Research, 93, 151-163. http://dx.doi.org/10.1016/S0079-6123(08)64570-7

[81] Antoniadis, E.A., Ko, C.H., Ralph, M.R. and McDonald, R.J. (2000) Circadian Rhythms, Aging and Memory. Behavioural Brain Research, 111, 25-37. http://dx.doi.org/10.1016/S0166-4328(00)00145-5

[82] Tevy, M.F., Giebultowicz, J., Pincus, Z., Mazzoccoli, G. and Vinciguerra, M. (2013) Aging Signaling Pathways and Circadian Clock-Dependent Metabolic Derangements. Trends in Endocrinology Metabolism, 24, 229-237. http://dx.doi.org/10.1016/j.tem.2012.12.002

[83] Jean-Louis, G., Kripke, D., Ancoli-Israel, S., Klauber, M.R., Sepulveda, R.S., Mowen, M.A., Assmus, J.D. and Langer, R.D. (2000) Circadian Sleep, Illumination, and Activity Patterns in Women: Influence of Aging and Time References. Physiology \& Behavior, 68, 347-352. http://dx.doi.org/10.1016/S0031-9384(99)00186-9

[84] Singletary, K.G. and Naidoo, N. (2011) Disease and Degeneration of Aging Neural Systems that Integrate Sleep Drive and Circadian Oscillations. Frontiers in Neurology, 2, 66. http://dx.doi.org/10.3389/fneur.2011.00066

[85] Yu, E.A. and Weaver, D.R. (2011) Disrupting the Circadian Clock: Gene-Specific Effects on Aging, Cancer, and Other Phenotypes. Aging, 3, 479-493.

[86] Antoch, M.P., Gorbacheva, V.Y., Vykhovanets, O., Toshkov, I.A., Kondratov, R.V., Kondratova, A.A., Lee, C. and Nikitin, A.Y. (2008) Disruption of the Circadian Clock Due to the Mutation Has Discrete Effects on Aging and Carcinogenesis. Cell Cycle, 7, 1197-1204. http://dx.doi.org/10.4161/cc.7.9.5886

[87] Wu, Y.H. and Swaab, D.F. (2007) Disturbance and Strategies for Reactivation of the Circadian Rhythm System in Aging Alzheimer's Disease. Sleep Medicine, 8, 623-636. http://dx.doi.org/10.1016/j.sleep.2006.11.010

[88] Harper, D.G., Volicer, L., Stopa, E.G., McKee, A.C., Nitta, M. and Satlin, A. (2005) Disturbance of Endogenous Circadian Rhythm in Aging and Alzheimer's Disease. American Journal of Geriatric Psychiatry, 13, 359-368. http://dx.doi.org/10.1097/00019442-200505000-00004

[89] Van Cauter, E., Plat, L., Leproult, R. and Copinschi, G. (1998) Alterations of Circadian Rhythmicity and Sleep in Aging: Endocrine Consequences. Hormone Research, 49, 147-152. http://dx.doi.org/10.1159/000023162

[90] Pierpaoli, W., Bulian, D., Dall’Ara, A., Marchetti, B., Gallo, F., Morale, M.C., Tirolo, C. and Testa, N. (1997) Circadian Melatonin and Young-to-Old Pineal Grafting Postpone Aging and Maintain Juvenile Conditions of Reproductive Functions in Mice and Rats. Experimental Gerontology, 32, 587-602. http://dx.doi.org/10.1016/S0531-5565(96)00163-5

[91] Zhang, Y., Kornhauser, J.M., Zee, P.C., Mayo, K.E., Takahashi, J.S. and Turek, F.W. (1996) Effects of Aging on Light-Induced Phase-Shifting of Circadian Behavior Rhythms, Fos Expression and CREB Phosphorylation in the Hamster Suprachiasmatic Nucleus. Neuroscience, 70, 951-961. http://dx.doi.org/10.1016/0306-4522(95)00408-4 


\section{Abbreviations}

ET, electron transfer; ROS, reactive oxygen species; RNS, reactive nitrogen species; OS, oxidative stress; NADPH, nicotinamide adenine dinucleotide phosphate-oxidase; SCN, suprachiasmatic nucleus; AT1, angiotensin II type 1; PER2, period circadian protein homolog 2; SNP, single nucleotide polymorphism; 5-HT, seratonoin receptor; NAT, N-acetyl transferase; Mel 1b, melatonin 1b; PKA, protein kinase A; ER alpha, estrogen receptor alpha; BP, bipolar disorder; RF-amide peptides, peptides with C-terminal arginine-phenylalanine amid; AD, Alzheimer's disease; REV-ERB alpha or NR1D1, nuclear receptor subfamily 1 proteins; AVP, arginine vasopressin. 
Scientific Research Publishing (SCIRP) is one of the largest Open Access journal publishers. It is currently publishing more than 200 open access, online, peer-reviewed journals covering a wide range of academic disciplines. SCIRP serves the worldwide academic communities and contributes to the progress and application of science with its publication.

Other selected journals from SCIRP are listed as below. Submit your manuscript to us via either submit@scirp.org or Online Submission Portal.
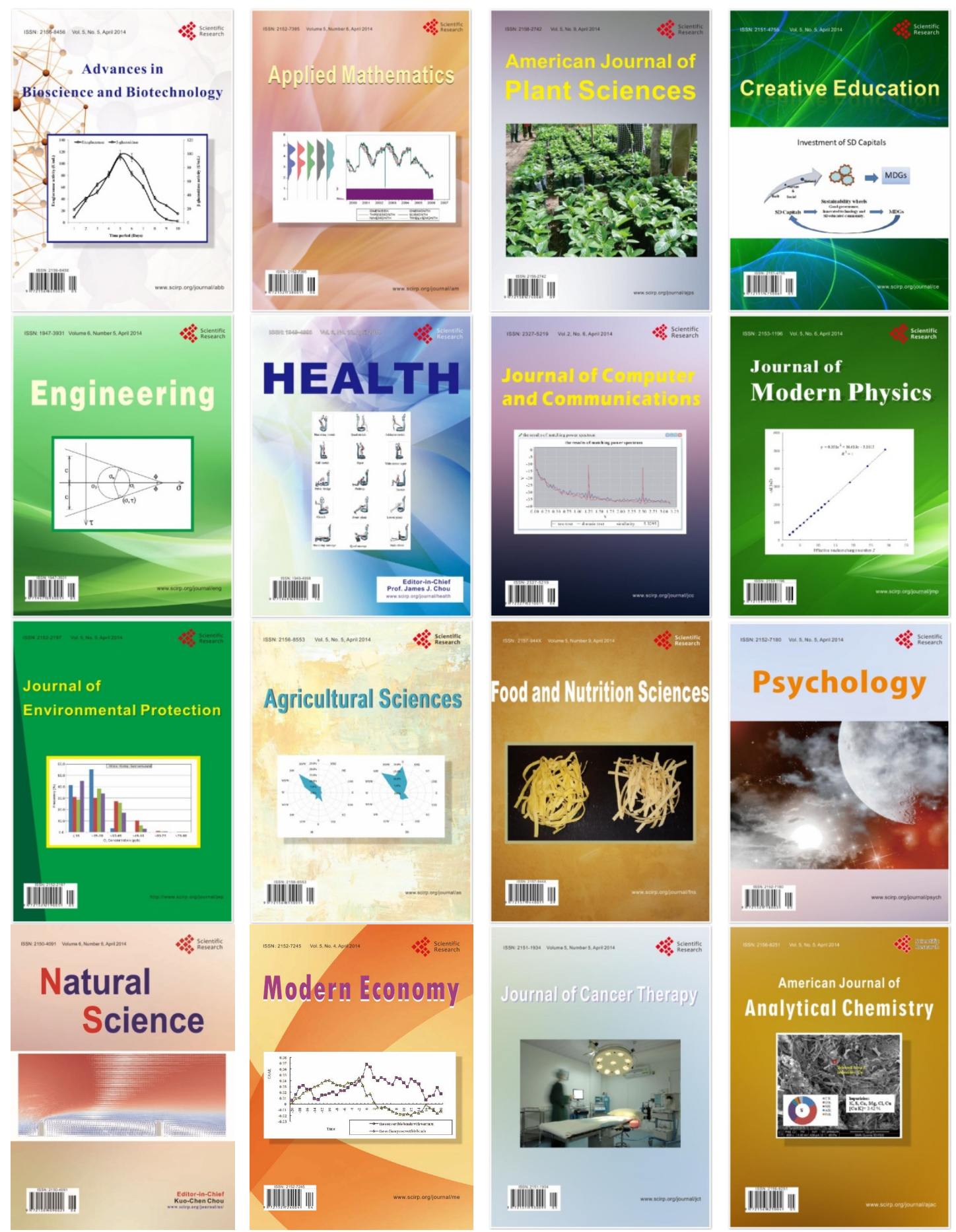